\title{
Intra-Arterial MSC Transplantation Restores Functional Capacity After Skeletal Muscle Trauma
}

\author{
Philipp von Roth ${ }^{*}{ }^{1,2}$, Georg N. Duda ${ }^{2,3}$, Piotr Radojewski ${ }^{1}$, Bernd Preininger ${ }^{1}$, Kristin Strohschein ${ }^{2}$, \\ Eric Röhner ${ }^{1}$, Carsten Perka ${ }^{1,2}$ and Tobias Winkler ${ }^{1}$
}

${ }^{I}$ Center for Musculoskeletal Surgery and Julius Wolff Institute, ${ }^{2}$ Berlin-Brandenburg School for Regenerative Therapies, ${ }^{3}$ Berlin-Brandenburg Center for Regenerative Therapies and Julius Wolff Institue, Charité - Universitätsmedizin Berlin, Free and Humboldt-University of Berlin, Germany

\begin{abstract}
Introduction: Skeletal muscle trauma leads to severe functional deficits, which cannot be addressed by current treatment options. Our group could show the efficacy of local transplantation of mesenchymal stroma cells (MSCs) for the treatment of injured muscles. While local application of MSCs has proven to be effective, we hypothesized that a selective intra-arterial transplantation would lead to a better distribution of the cells and so improved physiological recovery of muscle function.

Materials and Methodology: 18 female Sprague Dawley rats received an open crush trauma of the left soleus muscle. Autologous MSC were transduced using dsCOP-GFP and $2.5 \times 10^{6}$ cells were transplanted into the femoral artery of 9 animals one week after trauma. Control animals $(n=9)$ received a saline injection. Cell tracking, analysis of tissue fibrosis and muscle force measurements were performed after 3 weeks.

Results: Systemic MSC-therapy improved the muscle force significantly compared to control (fast twitch: $82.4 \%$, tetany: $61.6 \%, \mathrm{p}=0.02$ ). The histological analysis showed no differences in the quantity of fibrotic tissue. Histological examination revealed no cells in the traumatized muscle tissue 21 days after transplantation.

Conclusions: The present study demonstrated an effect of systemically administered MSCs in the treatment of skeletal muscle injuries. For possible future therapeutic approaches a systemic application of MSCs seems to present an alternative to a local administration. Such systemic treatment would be preferable since it allows functional improvement and possible cellular concentration at injury sites that are not easily accessible.
\end{abstract}

Keywords: Muscle, trauma, regeneration, stem cells, stroma cells, intra-arterial, systemic.

\section{INTRODUCTION}

Due to non-existent treatment options, skeletal muscle injuries and traumata pose a challenge to any current surgical strategy. Moreover, iatrogenic muscle trauma such as in orthopedic surgery impairs the functional outcome after treatment. Current surgical techniques focus on the reconstitution of biomechanical and anatomical competences of the bony structures at the expense of the muscular components. The lack of strategies to address muscle trauma and injury is probably one of the greatest remaining challenges in orthopedic surgery. The relevance of muscle deficits and scar tissue formation is widely underestimated. Scar formation and fatty muscle degeneration certainly remain the most outstanding challenges in regaining functional recovery and so new therapeutic strategies to regenerate functional muscle tissue are urgently needed.

Stem cell therapy is a promising tool to enhance regeneration processes. Lee et al. could induce long-term functional improvement following intravenous administration

*Address correspondence to this author at the Center for Musculoskeletal Surgery, Charité - Universitätsmedizin Berlin, Free and HumboldtUniversity of Berlin, Charitéplatz 1, D-10117 Berlin, Germany;

Tel: +49 - 30 - 450 659103; Fax: +49 - 30 - 450 522907;

E-mail: philipp.roth@charite.de of human neural stem cells in Huntington's disease [1]. Migration of intra-arterially injected stem cells into the brain after traumatic brain injury was reported [2].

Mesenchymal stroma cells (MSCs) have the potential to differentiate into mesenchymal lineages including bone, cartilage, tendon, fat, muscle and marrow stroma that support hematopoesis [3]. These characteristics make MSCs an ideal candidate for cell-based therapeutic strategies for tissue injuries for both local and systemic application.

In the field of myocardial regeneration, several clinical studies to enhance the myocardial function have already been performed. MSCs are thought to regenerate heart tissue more via paracrine effects such as stimulating angiogenesis and having immunmodulatory effects as opposed to via differentiation into myocytes and endothelial cells [4]. Nevertheless, systemic transplantation of bone-marrowderived MSCs following myocardial infarction led to preferential migration and colonization of the cells to the ischemic myocardium [5]. A consequence seems to be the reduction of the amount of fibrosis that is associated with muscle trauma [6].

For initial attempts to regenerate skeletal muscle, myoblasts were transplanted into a mouse model for muscular dystrophy [7]. Studies on MSCs and skeletal 
muscle regeneration are very rare. Natsu et al. transplanted MSCs seeded on a fibrin scaffold after laceration injury of the tibialis anterior muscle. After treatment the muscle force of the animals was almost completely recovered [8].

The mechanisms of action of multipotent cells for the regeneration of muscle remain unclear. While some suggest that differentiation into mature muscle tissue and fusion with regenerating muscle fibers is the main effect others suggest a paracrine stimulation of the intrinsic regeneration by the cells [9].

MSCs secrete a large variety of cytokines and growth factors, such as vascular endothelial growth factor (VEGF), Interleukin 1-beta (IL-1beta), platelet-derived growth factor (PDGF) and insulin like growth factor-1 (IGF-1) [10]. All of these factors are associated with both types of regeneration. The biological active factors can modify the local immune system, the local formation of fibrosis, influence the differentiation and proliferation of cells and decrease the amount of apoptosis [11, 12]. Onishi et al. observed the reduction of fibrous tissue after the injection of MSCconditioned medium following myocardial infarction. MSCs secrete matrix-metallo-proteases (MMP-1, -2, -8, -9, -13), which are secreted into the extracellular matrix. There these can digest collagen fibers and consequently inhibit the formation of fibrosis and allow the invasion of vessels. The development of fibrous tissue plays a crucial role in muscle regeneration since fibrous tissue can only transduce forces passively and this tissue is lost for a functional recovery. Gnecchi et al. postulated a paracrine mode of action after they found decreased fibrosis and apoptosis following injection of MSC-conditioned medium after myocardial infarction [13].

Our group is investigating the influence of MSCs on skeletal muscle after severe crush injury. A dose-response relationship of the quantity of locally transplanted MSCs and force enhancement was described earlier [14, 15]. Migration of MRI-tracked MSCs could be excluded and histological analyses revealed fusion events between existing myofibers but only to a limited extent [16].

While local therapy with MSCs has proven to be effective in skeletal muscle, a systemic application - like the one shown for myocardial infarction - in skeletal muscle trauma has never been analyzed to our knowledge. Therefore the aim of this study was to investigate the effect of an intraarterial transplantation of MSCs following an injury of skeletal muscle. Our hypothesis was that an intra-arterial transplantation of autologous mesenchymal stroma cells leads to an increase in functional regeneration in Sprague Dawley rats.

\section{MATERIALS AND METHODOLOGY}

\section{Animals}

Eighteen female Sprague Dawley rats (140 - $160 \mathrm{~g}$, Charles River, Sulzbach, Germany) were used in this study. Animals were kept at a constant temperature with free access to food and water. All animal experiments were carried out according to the policies and principles established by the Animal Welfare Act, the NIH Guide for Care and Use of Laboratory Animals and German national animal welfare guidelines. The study was approved by the German local legal authorities of Berlin (Reg.-Number G 251/06).

\section{Experimental Setup}

Bone marrow aspirations were taken from both tibiae of each animal under sterile conditions [14]. Two weeks later, the animals were separated into two groups (each $n=9$ ) and the left soleus muscle was bluntly crushed. Seven days later $2.5 \times 10^{6}$ MSCs were transplanted into the left femoral artery in Group 1. Group 2 served as a control group and received saline solution intra-arterial (i.a.). Three weeks after transplantation functional testing of the traumatized and contralateral healthy muscles was performed in vivo. Finally muscles were harvested for histological analysis.

\section{Bone Marrow Aspiration}

Bone marrow aspirations were performed as described before [14]. Briefly, anesthetized rats were subjected to tibial bone marrow aspirations and the marrow transferred into $10 \mathrm{ml}$ of Dulbecco's Modified Eagle Medium (DMEM, Gibco, Paisley, Great Britain) containing $10 \%$ fetal calf serum (Biochrome, Berlin, Germany) and 1\% PenicillinStreptomycin (Sigma, Taufkirchen, Germany), seeded in 75 $\mathrm{cm}^{2}$ cell culture flasks (Falcon, Heidelberg, Germany) and cultured at $37^{\circ} \mathrm{C}$ and $5 \% \mathrm{CO}_{2}$.

\section{Cell Culture}

Culture Medium was renewed every two days. At about $60 \%$ confluence, cells were split using $0.25 \%$ trypsin (Sigma, Germany) and cultivated until reaching passage three. Fluorescence-activated cell sorting (FACS) analysis was performed using the following antibodies: mouse $\alpha$-rat CD44 (Serotec, UK), mouse $\alpha$-rat CD45 and mouse $\alpha$-rat CD90 (both Acris Antibodies, Germany), mouse $\alpha$-rat CD73 and rat $\alpha$-mouse IgG (both BD Biosciences, Germany). The differentiation potential was tested for osteogenic and adipogenic differentiation potential (osteogenic medium: DMEM supplemented with $200 \mu \mathrm{M}$ ascorbic acid, $7 \mathrm{mM} \beta$ glycerophosphate and $0.01 \mu \mathrm{M}$ dexamethasone, adipogenic medium: DMEM supplemented with $1 \mu \mathrm{M}$ dexamethasone, $2 \mu \mathrm{M}$ insulin, $200 \mu \mathrm{M}$ indomethacine and $500 \mu \mathrm{M}$ isobutylmethylxanthine).

\section{Green Fluorescent Protein Transduction of MSC}

MSCs were seeded in $175 \mathrm{~cm}^{2}$ cell culture flasks at a density of 3000 cells $/ \mathrm{cm}^{2} 9$ days prior to transplantation. MSCs were transduced via a lentiviral system to insert dscopGFP (Lentivector Expressing Systems; System Biosciences, Mountain View, California, USA). Transduction was performed by adding virus, produced in Human Embryonic Kidney 293 cells (HEK), to the adherent MSCs. No transduction reagent was added.

\section{Skeletal Muscle Trauma}

Rats were anesthetized, the left leg was shaved, disinfected and an open crush injury of the left soleus muscles was performed [17]. The soleus muscles were crushed with a curved artery forceps (Aesculap, Tuttlingen, Germany) three times and distally to the insertion of the medial neurovascular bundle. An additional crushed injury was performed two times proximally of the neurovascular bundle, thereby preserving innervation and blood supply to 
the whole muscle structure. Standardization of the trauma was guaranteed by applying the forceps with constant pressure (closing pressure: $112 \pm 5.1 \mathrm{~N}$, data obtained from the material testing device Zwick 1455 (Zwick GmbH, Ulm, Germany)).

\section{MSC Transplantation and Sham Injections}

One week after the trauma MSCs were prepared, resuspended in $1 \mathrm{ml} 0.9 \%$ saline solution and stored on ice until further use. Rats were anesthetized, the left leg and inguinal region were shaved. A $2 \mathrm{~cm}$ incision was made longitudinally on the proximal thigh. The left inguinal vessels were prepared carefully, the femoral artery was exposed and embraced distally and proximally with sutures (Fig. 1). A 27 Gauge Microflon (BD Biosciences, San Jose, USA) was introduced into the femoral artery and the resuspended cells were infused during 1 minute. The femoral artery was closed with a patch of autologous fat fixed with Histoarcyl ${ }^{\circledR}$ glue (B. Braun Melsungen AG, Melsungen, Germany).

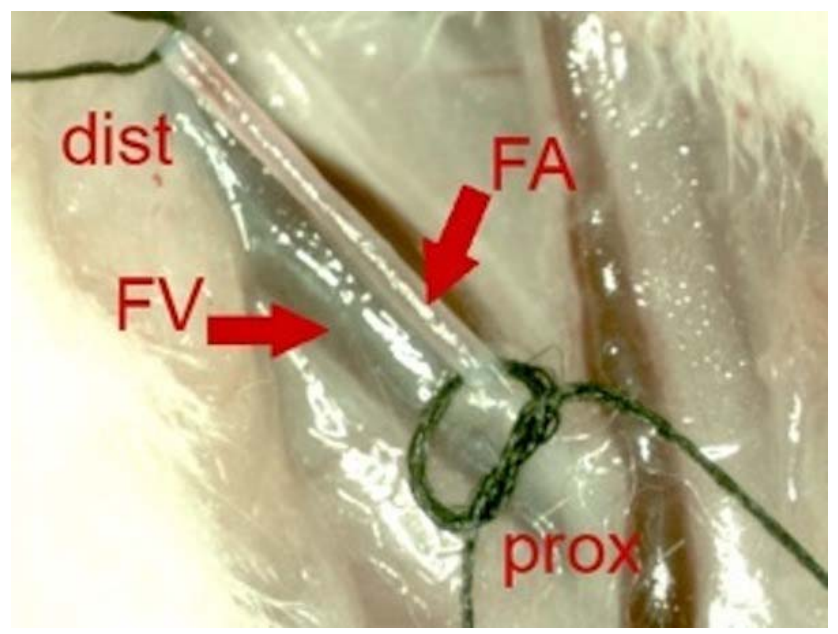

Fig. (1). Intraoperative photo before intra-arterial transplantation of MSC. Proximal femoral artery (FA) is embraced distally (dist) and proximally (prox) with suture wires. Parallel to the FA the femoral vain $(\mathrm{FV})$ can be identified.

\section{Biomechanical Evaluation}

The animals were anesthetized as described before [17]. The sciatic nerve and the soleus muscle were dissected and the tendon of the soleus muscle separated from the Achilles tendon. Muscles were tested serially. The lower extremity was fixated at the knee and ankle joint and the sciatic nerve was connected to a stimulator (Experimetria, Budapest, Hungary) $[18,19]$. The soleus muscle tendon was connected to the force transducer using vicryl suture. The computercontrolled muscle force measurement device applied a pretension of $0.15 \mathrm{~N}$ to the muscle and subsequently the sciatic nerve was stimulated bipolarly in two different modes (fast twitch: 5 pulses at $9 \mathrm{~mA} / 75 \mathrm{~Hz}$, duration $0.01 \mathrm{~s}$; tetany: 5 pulses at $9 \mathrm{~mA} / 75 \mathrm{~Hz}$, duration $3 \mathrm{~s}$; intervals $5 \mathrm{~s}$, respectively). The animals were sacrificed by intra-cardiac potassium injection.

\section{Histological Analysis}

Picro-sirius red staining was performed for collagenous connective tissues analysis. Cryo-preserved muscles were sectioned longitudinally $(6 \mu \mathrm{m})$. Total area of endo- and perimysial fibrosis was analyzed on images of whole longitudinal sections of the muscle by using a Leica DMRB light microscope (Leica, Wetzlar, Germany) equipped with an AxioCam MRc digital camera (Carl Zeiss, Gottingen, Germany).

\section{Statistical Analysis}

The median and standard error of the median were determined for each parameter. A statistical significance analysis was performed using the non-parametric MannWhitney-U test for independent samples for comparisons between the treatment and the control group. The level of significance was set to 0.05 . Left contraction forces were individually normalized to the uninjured right soleus muscle.

\section{RESULTS}

Twitch stimulation of the healthy right soleus muscles resulted in a contraction force of $0.52 \pm 0.14 \mathrm{~N}$. Forces of tetanic contraction in the uninjured muscles reached $0.98 \pm$ $0.27 \mathrm{~N}$. Maximum contraction forces after twitch stimulation of traumatized muscles were reduced to $0.43 \pm 0.08 \mathrm{~N}$. Tetanic stimulation revealed a reduction of the maximum contraction capacity to $0.52 \pm 0.08 \mathrm{~N}$ (Group 2). These results represent a decrease of muscle force of $17.3 \pm 6 \%$ after fast twitch and $46.9 \pm 16 \%$ after tetanic stimulation due to the trauma.

The intra-arterial MSC-transplantation improved the muscle force of the injured soleus significantly compared to the control (fast twitch: $82.4 \pm 30 \%, p=0.02$, tetany: $58.9 \pm$ $13 \%, \mathrm{p}=0.02$; Fig. 2).

The histomorphometric analysis showed a quantity of fibrotic tissue of $27.3 \pm 8.6 \%$ of cell treated soleus muscles and of $28.5 \pm 7.0 \%(p=0.38)$ in sham treated muscles. GFP positive MSC could not be found in histologic analysis of soleus muscle.

\section{DISCUSSION}

The aim of this study was to analyze the effect and feasibility of an intra-arterial mesenchymal stroma cell therapy for treating skeletal muscle injuries. We hypothesized that an intra-arterial transplantation of autologous mesenchymal stroma cells leads to an increase in functional regeneration. However, the cells could not be identified in the muscle tissue three weeks after transplantation.

Regeneration of muscle tissue follows an identical pattern independent of the trauma. Basically, the process of regeneration is induced via an inflammatory response which itself represents the first phase of this process (Phase I). Satellite cells proliferate and migrate into the center of myofibers (Phase II). The process is terminated by the formation of fibrosis (Phase III) [20]. Kääriäinen et al. reported the formation of scar tissue just 2 days after trauma [21].

Previously it was shown that MSCs can secrete matrixmetallo-proteases (MMP). MSC transplantation reduced the formation of fibrosis and increased contractility in cardiac muscle. Our histological analysis showed no reduction of fibrosis following intra-arterial transplantation. These results 


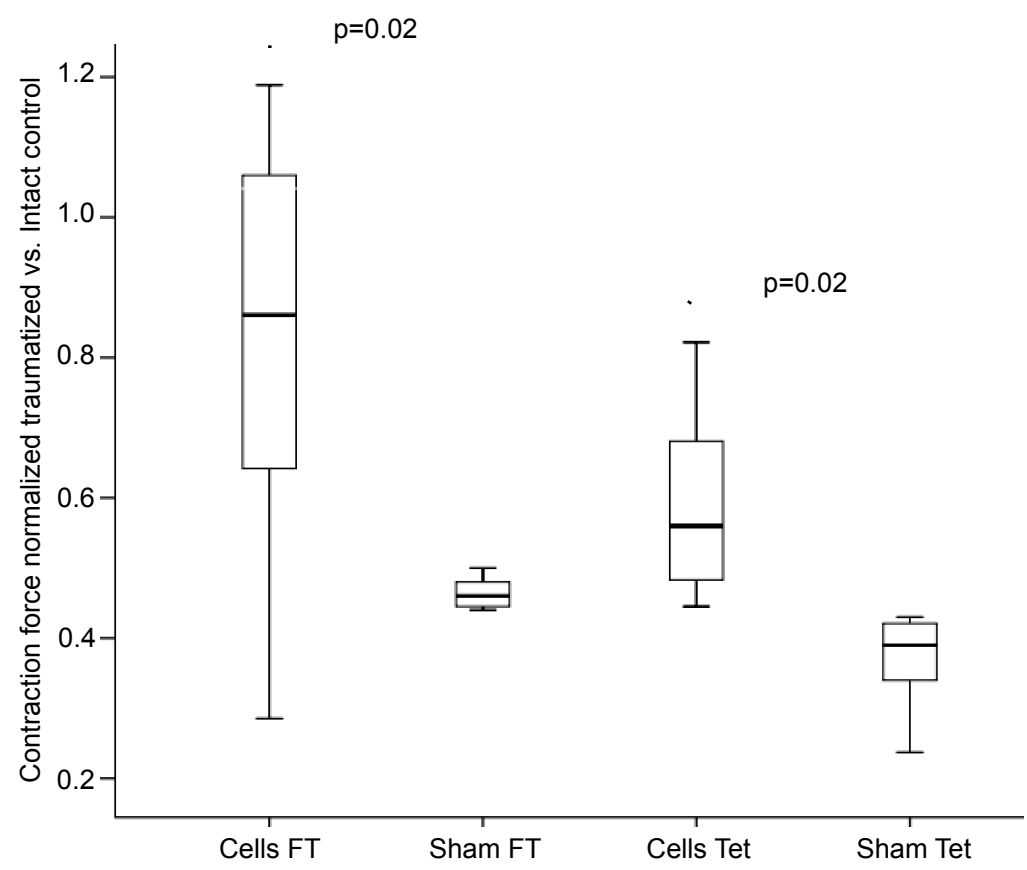

Fig. (2). Fast Twitch (FT) and tetanic (Tet) contraction forces of control animals (Sham) and after intra-arterial transplantation (Cells) of $2,5 \times 10^{6}$ autologous MSC.

are in accordance with our previous investigations where no influence on the formation of fibrous tissue was found [1416]. Vaittinen et al. did show the formation of fibrous tissue after laceration. The resulting scar tissue was even detectable after 12 months and the separated muscles were connected via myotendinous junctions without showing definite fusion [21]. Previously it was assumed that the reduction of fibrous tissue would result in a higher contraction capability. If the results of our present and previous studies are taken into consideration then it can be hypothesized that the fibrous tissue might be important for force transduction. An uncontrolled degradation of the fibrosis might result in an instable biomechanical situation of soleus muscle. The presence of fibrous tissue, on the other hand, does not appear to be detrimental for a sufficient contraction force development.

Surprisingly, transplanted cells could not be found in the traumatized muscle tissue indicating an effect of the cells by the secretion of soluble factors. Meng et al. transplanted musclederived stem cells systemically into dystrophic mice and were also unable to detect cells within the target muscle [22]. A functional assessment was not performed and so a possible paracrine effect could not be excluded. Earlier investigations by our group after local transplantations showed fusion events of transplanted MSCs and regenerating myofibers but only to a very limited extent. The observed increase in functional regeneration could not be attributed to these events alone [16]. As we also observed an increased functional recovery following systemic transplantation, we assume a mode of action via soluble factors of the transplanted MSCs.

A possible mode of action of MSCs is via their immunomodulatory abilities. In vitro and in vivo investigations showed an immunosuppressive effect of MSCs on T-cells [23, 24]. MSCs were able to inhibit the proliferation of T-cells, which were stimulated by allogeneic lymphocytes. It has also been demonstrated that MSCs inhibit the proliferation, maturation and activation of dendritic cells $[25,26]$ and reduce the proliferation, cytokine secretion and cytotoxicity of natural killer cells [27]. MSCs also reduce the effect of necrosis/apoptosis-inducing cells and have cytoprotective effect. Further investigations have to address a possible effect through soluble factors of MSC on the attenuation of the intramuscular immune response.

\section{CONCLUSION}

To conclude, a systemic transplantation of bone marrowderived cells for the improved regeneration of injured musculature appears to be feasible. A major advantage of an intra-arterial transplantation of cells is that they can be performed highly selectively in patients via interventional radiographic catheters. This opens up the possibility of treating skeletal muscle injuries in regions that are normally not easily accessible.

\section{AUTHORS' CONTRIBUTIONS}

Philipp von Roth: Design of the study, animal surgery, and preparation of the manuscript.

Georg N. Duda: Design of the study, analysis and interpretation of data and preparation of manuscript.

Piotr Radojewski: In vitro preparation of cells, muscle force measurements.

Bernd Preininger: Analysis and interpretation of data, preparation of the manuscript.

Kristin Strohschein: Revision of the manuscript.

Eric Röhner: Analysis and interpretation of data, preparation of the manuscript.

Carsten Perka: Design of the study analysis and interpretation of data and preparation of manuscript. 
Tobias Winkler: Design of the study, animal surgery, and preparation of the manuscript.

\section{ACKNOWLEDGEMENTS}

We like to thank Jasmine Müller for her excellent technical assistance. Monetary support was received from a kick-off grant of the Berlin-Brandenburg Center for Regenerative Therapies.

\section{CONFLICT OF INTERESTS}

Authors confirm that this article content has no conflicts of interest.

$$
\begin{aligned}
& \text { ABBREVIATIONS } \\
& \text { dist }=\text { Distally } \\
& \text { DMEM = Dulbecco's Modified Eagle Medium } \\
& \text { FT }=\text { Fast twitch } \\
& \text { FACS = Fluorescence-activated cell sorting } \\
& \text { FA }=\text { Femoral artery } \\
& \mathrm{FV} \quad=\text { Femoral vain } \\
& \text { HEK = Human Embryonic Kidney } 293 \text { cells } \\
& \text { IGF-1 = Insulin like growth factor-1 } \\
& \text { IL-1beta }=\text { Interleukin 1-beta } \\
& \text { i.a. }=\text { Intra-arterial } \\
& \text { MMP = Matrix-metallo-proteases } \\
& \text { MSCs = Mesenchymal stroma cells } \\
& \text { PDGF }=\text { Platelet derived growth factor } \\
& \text { prox }=\text { Proximally } \\
& \text { Tet }=\text { Tetany } \\
& \text { VEGF }=\text { vascular endothelial growth factor }
\end{aligned}
$$

\section{REFERENCES}

[1] Lee ST, Park JE, Lee K, et al. Noninvasive method of immortalized neural stem-like cell transplantation in an experimental model of Huntington's disease. J Neurosci Methods 2006; 152(1-2): 250-4.

[2] Mahmood A, Lu D, Chopp M. Intravenous administration of marrow stromal cells (MSCs) increases the expression of growth factors in rat brain after traumatic brain injury. J Neurotrauma 2004; 21(1): 33-9.

[3] Pittenger MF, Mackay AM, Beck SC, et al. Multilineage potential of adult human mesenchymal stem cells. Science 1999;284(5411): 143-7.

[4] Nagaya N, Fujii $\mathrm{T}$, Iwase $\mathrm{T}$, et al. Intravenous administration of mesenchymal stem cells improves cardiac function in rats with acute myocardial infarction through angiogenesis and myogenesis. Am J Physiol Heart Circ Physiol 2004; 287(6): H2670-6.

[5] Barbash IM, Chouraqui P, Baron J, et al. Systemic delivery of bone marrow-derived mesenchymal stem cells to the infarcted myocardium: feasibility, cell migration, and body distribution. Circulation 2003; 108(7): 863-8.

[6] Bartunek J, Vanderheyden M, Vandekerckhove B, et al. Intracoronary injection of CD133-positive enriched bone marrow progenitor cells promotes cardiac recovery after recent myocardial infarction: feasibility and safety. Circulation 2005; 112 (9 Suppl): I178-83.
[7] Kinoshita I, Vilquin JT, Guerette B, Asselin I, Roy R, Tremblay JP. Very efficient myoblast allotransplantation in mice under FK506 immunosuppression. Muscle Nerve 1994; 17(12): 1407-15.

[8] Natsu K, Ochi M, Mochizuki Y, Hachisuka H, Yanada S, Yasunaga Y. Allogeneic bone marrow-derived mesenchymal stromal cells promote the regeneration of injured skeletal muscle without differentiation into myofibers. Tissue Eng 2004; 10(7-8): 1093-112.

[9] Wang J, Liao L, Tan J. Mesenchymal-stem-cell-based experimental and clinical trials: current status and open questions. Expert Opin Biol Ther 2011; 11(7): 893-909.

[10] Takahashi M, Li T, Suzuki R, et al. Cytokines produced by bone marrow cells can contribute to functional improvement of the infarcted heart by protecting cardiomyocytes from ischemic injury. Am J Physiol Heart Circ Physiol 2006; 291(2): H886-93.

[11] Mangi AA, Noiseux N, Kong D, et al. Mesenchymal stem cells modified with Akt prevent remodeling and restore performance of infarcted hearts. Nat Med 2003; 9(9): 1195-201.

[12] Tang YL, Zhao Q, Zhang YC, et al. Autologous mesenchymal stem cell transplantation induce VEGF and neovascularization in ischemic myocardium. Regul Pept 2004; 117(1): 3-10.

[13] Gnecchi M, Zhang Z, Ni A, Dzau VJ. Paracrine mechanisms in adult stem cell signaling and therapy. Circ Res 2008; 103(11): 1204-19.

[14] Matziolis G, Winkler T, Schaser K, et al. Autologous bone marrowderived cells enhance muscle strength following skeletal muscle crush injury in rats. Tissue Eng 2006; 12(2): 361-7.

[15] Winkler T, von Roth P, Matziolis G, Mehta M, Perka C, Duda GN Dose-response relationship of mesenchymal stem cell transplantation and functional regeneration after severe skeletal muscle injury in rats. Tissue Eng Part A 2009; 15(3): 487-92.

[16] Winkler $\mathrm{T}$, von Roth $\mathrm{P}$, Schuman $\mathrm{M}$, et al. In vivo visualization of locally transplanted mesenchymal stem cells in the severely injured muscle in rats. Tissue Eng Part A 2008; 14(7): 1149-60.

[17] Winkler T, von Roth P, Matziolis G, et al. Time course of skeletal muscle regeneration after severe trauma. Acta Orthop 2011; 82(1): 10211 .

[18] Racz IB, Illyes G, Sarkadi L, Hamar J. The functional and morphological damage of ischemic reperfused skeletal muscle. Eur Surg Res 1997; 29(4): 254-63.

[19] Fish JS, McKee NH, Pynn BR, Kuzon WM Jr, Plyley MJ. Isometric contractile function recovery following tourniquet ischemia. J Surg Res 1989; 47(4): 365-70.

[20] Quintero AJ, Wright VJ, Fu FH, Huard J. Stem cells for the treatment of skeletal muscle injury. Clin Sports Med 2009;28(1): 1-11.

[21] Vaittinen S, Hurme T, Rantanen J, Kalimo H. Transected myofibres may remain permanently divided in two parts. Neuromuscul Disord 2002; 12(6): 584-7.

[22] Meng J, Adkin CF, Xu SW, Muntoni F, Morgan JE. Contribution of human muscle-derived cells to skeletal muscle regeneration in dystrophic host mice. PLoS ONE 2011; 6(3): e17454.

[23] Ramasamy R, Tong CK, Seow HF, Vidyadaran S, Dazzi F. The immunosuppressive effects of human bone marrow-derived mesenchymal stem cells target $\mathrm{T}$ cell proliferation but not its effector function. Cell Immunol 2008; 251(2): 131-6.

[24] Devine SM, Cobbs C, Jennings M, Bartholomew A, Hoffman R Mesenchymal stem cells distribute to a wide range of tissues following systemic infusion into nonhuman primates. Blood 2003; 101(8): 29993001.

[25] Jung YJ, Ju SY, Yoo ES, et al. MSC-DC interactions: MSC inhibit maturation and migration of BM-derived DC. Cytotherapy 2007; 9(5): 451-8.

[26] Zhang W, Ge W, Li C, et al. Effects of mesenchymal stem cells on differentiation, maturation, and function of human monocyte-derived dendritic cells. Stem Cells Dev 2004; 13(3): 263-71.

[27] Rasmusson I, Ringden O, Sundberg B, Le Blanc K. Mesenchymal stem cells inhibit the formation of cytotoxic T lymphocytes, but not activated cytotoxic T lymphocytes or natural killer cells. Transplantation 2003; 76(8): 1208-13 